\title{
On the phase-noise and phase-error performances of multiphase LC CMOS VCOs
}

\section{Andreani, Pietro; Wang, Xiaoyan}

Published in:

I E E E Journal of Solid State Circuits

Link to article, DOI:

10.1109/JSSC.2004.835828

Publication date:

2004

Document Version

Publisher's PDF, also known as Version of record

Link back to DTU Orbit

Citation $(A P A)$ :

Andreani, P., \& Wang, X. (2004). On the phase-noise and phase-error performances of multiphase LC CMOS VCOs. I E E E Journal of Solid State Circuits, 39(11), 1883-1893. https://doi.org/10.1109/JSSC.2004.835828

\section{General rights}

Copyright and moral rights for the publications made accessible in the public portal are retained by the authors and/or other copyright owners and it is a condition of accessing publications that users recognise and abide by the legal requirements associated with these rights.

- Users may download and print one copy of any publication from the public portal for the purpose of private study or research.

- You may not further distribute the material or use it for any profit-making activity or commercial gain

- You may freely distribute the URL identifying the publication in the public portal 


\title{
On the Phase-Noise and Phase-Error Performances of Multiphase $L C$ CMOS VCOs
}

\author{
Pietro Andreani, Member, IEEE, and Xiaoyan Wang
}

\begin{abstract}
This paper presents an analysis of phase noise in multiphase $L C$ oscillators, and measurement results for several CMOS quadrature-voltage-controlled-oscillators (QVCOs) working in the 2-GHz frequency range. The phase noise data for a so-called BS-QVCO $(-140 \mathrm{dBc} / \mathrm{Hz}$ or less at $3 \mathrm{MHz}$ frequency offset from the carrier, for a power consumption of $20.8 \mathrm{~mW}$ and a figure-of-merit of $184 \mathrm{dBc} / \mathrm{Hz}$ ) show that phase noise performances are close to the previously derived limits. A systematic cause of departure from ideal quadrature between QVCO signals is also analyzed and measured experimentally, and a compact $L C$-tank layout that removes this source of phase error is proposed. A TS-QVCO designed with this technique shows a phase-noise figure-of-merit improvement of $4 \mathrm{~dB}$, compared to a previous implementation. The measured equivalent phase error for all QVCOs is between $0.6^{\circ}$ and $1^{\circ}$.
\end{abstract}

Index Terms-CMOS, phase noise, quadrature signal generation, RF, voltage-controlled oscillators (VCOs).

\section{INTRODUCTION}

$\mathbf{T}$ HE problem of generating quadrature signals has recently attracted a great amount of interest, spurred by the key role played by such signals in low-IF and direct-conversion receivers. A power-efficient approach is by direct quadrature generation in so-called quadrature voltage-controlled oscillators (QVCOs), most often employing $L C$ tanks, because of the stringent requirements on the maximum acceptable phase noise in modern telecommunication standards.

The present paper deals primarily with the phase noise performances that can be expected from multiphase $L C$ oscillators in general, and in particular from the QVCO architectures presented in [1] and [2]. Since the noise performances in the $1 / f^{3}$ region for the design in [1] have been discussed at length in [3], we shall focus here on the phase noise in the $1 / f^{2}$ region, i.e., where the phase noise is caused by white noise sources.

General results will be derived in Section II by employing Hajimiri's simple and elegant theory of phase noise [4], centered upon the concept of impulse sensitivity function (ISF, with symbol $\Gamma$ ). Although the ISF approach has been superseded by analog simulators like spectreRF (based on Kärtner's mathematical treatment [5], [6]) as a numerical analysis tool, the closed-form formulation of the ISF will be used here to arrive to simple formulas for the phase noise generated by the noise sources in the $L C$ tanks. These formulas will enable the straightforward comparison between the performances of different mul-

Manuscript received January 8, 2004; revised July 7, 2004.

The authors are with the Center for Physical Electronics, Ørsted DTU, Technical University of Denmark, DK-2800 Lyngby, Denmark (e-mail: pa@oersted.dtu.dk).

Digital Object Identifier 10.1109/JSSC.2004.835828 tiphase oscillators, and even between oscillators generating different numbers of phases. SpectreRF phase-noise simulations will be illustrated in Section III, and compared to the results obtained in Section II; these comparisons will disclose the outstanding performances of which the QVCOs in [1] and [2] are capable. Section IV will present an analysis of the systematic phase error induced by a parasitic magnetic coupling between different $L C$ tanks, together with the technique adopted to avoid this effect. Section V will present both the excellent measurement results on two different QVCO prototypes, and the experimental evidence for the phase-error mechanism discussed in Section IV. Finally, Section VI will summarize the paper, Appendix A will prove the validity of the mathematical approach followed in Section II, and Appendix B will discuss an important property of phase noise.

\section{NOISE AND PHASE NOISE}

In this section, we focus on the following question: how does the phase noise of a multiphase oscillator relate to the phase noise of a single-phase oscillator, when both employ the same $L C \operatorname{tank}(\mathrm{s})$ ?

In order to provide an answer, we will make use of (34) in [4], reproduced in (1) for convenience:

$$
\Delta \phi_{i}=\frac{2 \pi}{T} \cdot \frac{\Delta q_{i}}{C_{i}} \cdot \frac{\dot{v}_{i}}{|\dot{\vec{v}}|^{2}}
$$

where $\Delta \phi_{i}$ is the excess phase caused by a current impulse at node $i$, which results in an excess charge $\Delta q_{i}$ on capacitor $C_{i}$, $T$ is the period of the oscillation, $\dot{v}_{i}$ is the derivative of the $i$ th node voltage, and $|\dot{\vec{v}}|$ is the norm of the first derivative of the waveform vector. It should be noted the (1) is not correct in general, ${ }^{1}$ since it assumes that noise perpendicular to the state-space trajectory of the oscillator does not generate any phase noise. ${ }^{2}$ However, we will show in Appendix A that (1) is in fact a very good approximation for the exact value of $\Delta \phi_{i}$, if the state equations associated to the currents flowing into the tank inductances are normalized with the impedance factor $\sqrt{L / C}$, where $L$ and $C$ are the inductance and capacitance for each tank. Assuming

\footnotetext{
${ }^{1}$ It is worth mentioning that the particular form of $\Delta \phi_{i}$ given by (1) only played a minor role in Hajimiri's theory [4]. In that work, the favored way of determining $\Delta \phi_{i}$ was through a series of time-shifted transient simulations. While this approach does yield results that are valid in general, such results are numerical in nature, and lack therefore the kind of universality possessed by symbolic closed-form expressions.

${ }^{2}$ The reader interested in these issues is referred to the papers by Kärtner [5], [6], as well as by Demir et al. [7]. Specific examples of systems where (1) yields wrong results are discussed in [8], and at the end of Appendix A in the present work. Useful information can be found in the Cadence spectreRF manual as well [9].
} 
that the voltage across $C$ is sinusoidal, which is a very good approximation even for moderate values of the quality factor $Q$ of the $L C$ tanks, a second consequence of this normalization (see again Appendix A) is that the voltage across $C$ and the voltage associated to the current flowing into $L$ have the same peak amplitude $A$, and are in quadrature to each other. If we now use the fact that in a well-designed multiphase $L C$ oscillator the voltages at the various tanks have the same amplitude and well-defined phase relations, and that signal amplitudes at other (parasitic) nodes in the oscillator are much smaller than $A$, we can rewrite (1) as

$$
\Delta \phi_{i} \approx \frac{2 \pi}{T} \cdot \frac{\Delta q_{i}}{C_{i}} \cdot \frac{A \omega_{0} \sin \left(\omega_{0} t\right)}{A^{2} \omega_{0}^{2} \cdot S}=\frac{\Delta q_{i}}{q_{\max }} \cdot \frac{\sin \left(\omega_{0} t\right)}{S}
$$

where $q_{\max }$ is the maximum signal charge stored in $C_{i}$ across an oscillation period, and $S$ is given by

$$
\begin{aligned}
S= & \left.\sin ^{2}\left(\omega_{0} t+\Phi_{0}\right)+\cos ^{2}\left(\omega_{0} t+\Phi_{0}\right)\right] \\
& +\cdots+\left[\sin ^{2}\left(\omega_{0} t+\Phi_{n}\right)+\cos ^{2}\left(\omega_{0} t+\Phi_{n}\right)\right]=n,
\end{aligned}
$$

with $n$ the number of tanks in the oscillator, and $\Phi_{j}$ the phase offset between the voltage at node $j$ and the voltage at node $i$. This result was already stated in [4] for $n=1$, and used in subsequent works.

According to the ISF theory, and employing (2) and (3), we obtain in general

$$
\Gamma=\frac{\sin \left(\omega_{0} t\right)}{n}
$$

resulting in an rms value of

$$
\Gamma_{\mathrm{rms}}^{2}=\frac{1}{2 n^{2}}
$$

to be used ${ }^{3}$ in the well-known expression [4], [11] for phase noise

$$
\mathcal{L}(\Delta \omega)=10 \log \left(\frac{\Gamma_{\mathrm{rms}}^{2}}{q_{\max }^{2}} \cdot \frac{\overline{\overline{i_{n}^{2}}}}{2 \Delta \omega^{2}}\right)
$$

where $\mathcal{L}(\Delta \omega)$ is the phase noise at the offset frequency $\Delta \omega$, generated by the noise power density $\overline{i_{n}^{2}} / \Delta f$ of the noise current source between node $i$ and ground. In the present case, $\overline{i_{n}^{2}}$ is the noise current associated to the parallel resistance $R_{\mathrm{tank}}$ of the tank, given by

$$
\overline{i_{n}^{2}}=4 k_{B} T \frac{1}{R_{\mathrm{tank}}} \Delta f
$$

where $k_{B}$ is Boltzman's constant, and $T$ the absolute temperature. We can obtain an expression for the noise power $N_{\mathcal{L}}$ responsible for this phase noise, by rewriting $\mathcal{L}$ as a noise-tosignal ratio:

$$
\mathcal{L}(\Delta \omega)=10 \log \left(\frac{N_{\mathcal{L}}}{A^{2} / 2}\right)
$$

\footnotetext{
${ }^{3} \mathrm{~A}$ dependence of $\Gamma_{\text {rms }}$ on $n$ in ring oscillators was observed, through numerical ISF simulations, in [10].
}

The above formulation of $\mathcal{L}$ is valuable in practice, since spectreRF expresses the phase noise data in terms of $N_{\mathcal{L}}$ and signal power. Noticing that $q_{\max }^{2}=\left(C_{i} A\right)^{2}=(C A)^{2}$, and using (6)-(8), we obtain

$$
N_{\mathcal{L}}(\Delta \omega)=\frac{\Gamma_{\mathrm{rmm}}^{2} \overline{i_{n}^{2}} / \Delta f}{4 C^{2} \Delta \omega^{2}}
$$

which shows that, when (5) holds, $N_{\mathcal{L}}$ is the same in all oscillators built around the same tank, i.e., $N_{\mathcal{L}}$ is independent of the different energy restoration mechanisms at work in different oscillators. It should be appreciated, though, that $N_{\mathcal{L}}$ depends on the node (or differential nodes) at which it is measured, while phase noise, remarkably, is independent of this choice (see, e.g., [12]; an intuitive proof of this statement is presented in Appendix B).

While (5) and (6) show that the presence of $n$ tanks reduces the phase noise due to a single noise source by a factor $n^{2}$, compared to the case of a single tank, there are now $n$ current noise sources instead of only one. These noise sources are all uncorrelated and equal in power, and using the above mentioned fact that phase noise is independent of the node where it is measured, we find that the total phase noise due to such noise sources is $n$ times the one given by (6). This result, however, assumes that $q_{\max }$ is the same in both cases, which is equivalent to assuming that the signal amplitude at each tank in the multiphase case is the same as in the single-phase case (this is not true in general [13], see Section II-A). If we now also assume that the other noise sources in the oscillator scale in the same proportion, the overall phase noise in the multiphase oscillator is $n$ times lower than in the single-phase case:

$$
\mathcal{L}_{n}(\Delta \omega)=\mathcal{L}_{1}(\Delta \omega)-10 \log (n) .
$$

However, it is clear that an oscillator with $n$ tanks draws (at least) $n$ times the current needed by the single-tank oscillator, if we want to have the same voltage amplitudes in both cases. Therefore, we can conclude that, under the above (ideal) assumptions, a multiphase oscillator displays a phase noise normalized to the power consumption that is independent of the number of phases generated. ${ }^{4}$

\section{A. Q Degradation and Other Nonideal Effects}

The previous analysis is ideal in the sense that it does not take into account various effects that might have a nonnegligible, or even dominant impact on the actual phase noise performances.

The severest problem is caused by the coupling between the tanks in a multiphase oscillator. It is well-known that when two differential $L C$-tank oscillators are coupled to generate quadrature phases, the phase noise performance may be easily destroyed by two mechanisms: the reduction of the effective $Q$ of the tanks, when the current flowing in each tank is not in phase with the tank voltage [13], and the additional noise generated by the coupling devices [3]. A remedy to both is to decrease the coupling strength between the two differential oscillators, at the

\footnotetext{
${ }^{4}$ The results in this section may be compared to those obtained by van der Tang et al. [13] with a linear analysis of the multiphase $L C$ oscillator. In particular, the result contained in (10) was also derived in the cited work, albeit in a somewhat different form.
} 

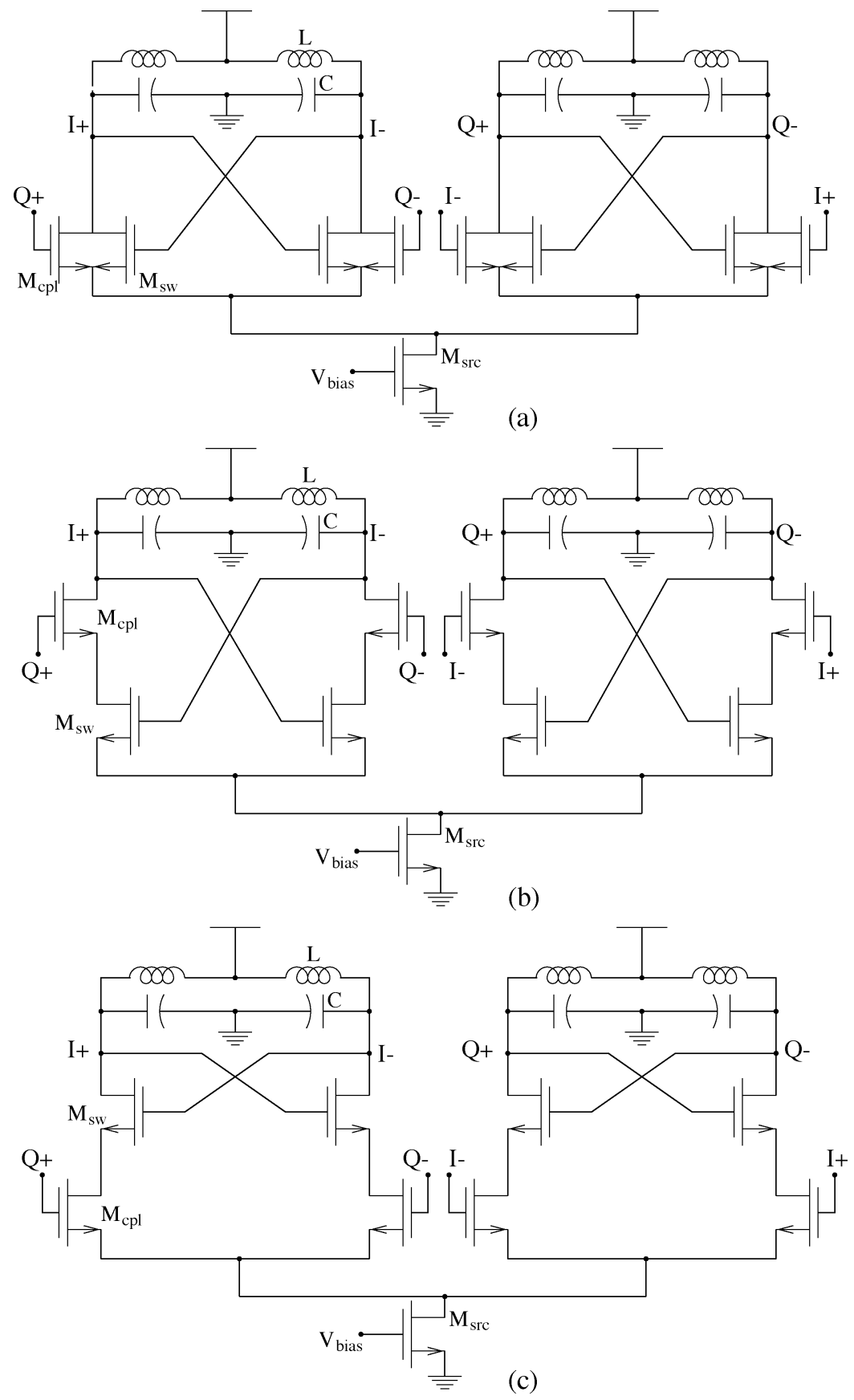

Fig. 1. Schematic views of the a) P-QVCO; b) TS-QVCO; c) BS-QVCO.

expense of a higher phase error between the phases for the same level of component mismatch [3]; in another approach, phase shifters may be introduced between the oscillators, in order to align current and voltage in each tank [13]. Yet another issue is that contributions to phase noise from parasitic nodes, such as the common source of the tail-biased differential oscillator, might have different weights when $n$ varies.

\section{Phase-Noise Simulations}

Several simulations were run with spectreRF, in order to assess the performances of three different $L C$ QVCO designs, all based on the mutual coupling of two differential $L C$ oscillators: the "parallel" QVCO in Fig. 1(a) [14] (P-QVCO, so-called because the transistors coupling the two differential oscillators are in parallel with the differential oscillators ${ }^{5}$ ), the "series" QVCO in Fig. 1(b) [1] (here to be referred to as top-series QVCO, TS-QVCO, since the coupling transistors are in series with the switch transistors, and placed above them), and the bottom-series QVCO of Fig. 1(c) [2] (BS-QVCO). Besides comparing these QVCOs with one another, it is natural to compare them with the differential oscillator they are based upon, and which is

${ }^{5}$ The original implementation of the P-QVCO had separated tail currents, while a unique tail current was proposed in [15]. This solution is adopted here for all three QVCOs examined, since it leads to (marginally) better phase noise performances. 
TABLE I

Simulated NoISE POWER CONTRIBUTIONS (IN $V_{\mathrm{rms}}^{2} / H z$ ) AND PHASE NoISE AT $3 \mathrm{MHz}$ FREQUENCY OFFSET FROM THE 2-GHz FundAMENTAL FOR THE DiFFERENTIAL (NONQUADRATURE) $L C$-TANK VCO $\left(Q_{\mathrm{tank}}=10\right.$ $\left.I_{\mathrm{BIAS}}=2 \times 4 \mathrm{~mA}\right)$

\begin{tabular}{lrr}
\hline \hline \multicolumn{3}{c}{ Differential VCO } \\
\hline $\mathrm{R}_{\text {tank }}$ & $2 \times 4.16 \cdot 10^{-16}$ & $59.8 \%$ \\
$\mathrm{M}_{\text {sw }}$ & $2 \times 2.80 \cdot 10^{-16}$ & $40.2 \%$ \\
Oscillation amplitude & $820 \mathrm{mV}_{\mathrm{p}}$ \\
Phase noise & $-143.8 \mathrm{dBc} / \mathrm{Hz}$ \\
\hline \hline
\end{tabular}

known to yield excellent performances in terms of phase-noise figure-of-merit (FoM), defined as

$$
\operatorname{FoM}(\Delta \omega)=-\mathcal{L}(\Delta \omega)+20 \log \left(\frac{\omega_{0}}{\Delta \omega}\right)-10 \log (P)
$$

where $\omega_{0}$ is the angular frequency of oscillation, and $P$ is the power (in $\mathrm{mW}$ ) consumed by the oscillator. For all these oscillators the inductance in each tank was $1.3 \mathrm{nH}$, the tank $Q$ was 10 (losses in the form of a parallel tank resistance $R_{\mathrm{tank}}$ of 163 $\Omega$ ), the oscillation frequency was fixed to $2 \mathrm{GHz}$, and the bias current $I_{\text {bias }}$ was $4 \mathrm{~mA}$ for each tank. In order not to bind the results to a specific technology and to a specific MOS model, the original bsim $3 \mathrm{v} .3$ model for the $0.35 \mu \mathrm{m}$ CMOS process used was modified in an ideal sense, by removing both the electron velocity saturation effect and the electron mobility reduction induced by the electric field normal to the MOS channel. Further, bias currents was supplied by ideal current sources, with a $100 \mathrm{fF}$ parasitic tail capacitance for each differential oscillator. These measures should be enough to make the behavior of the QVCOs largely process-independent, so that the results presented in the following should have a more general validity and be easily replicable.

In all simulations, the phase noise was measured in a singleended fashion, i.e., between a tank output and ground, which enables a direct comparison of the various $N_{\mathcal{L}}$ contributions in different oscillators (to repeat, the phase noise itself is independent of the node or nodes at which it is measured).

The simulation results for the differential VCO are summarized in Table I; the tank resistances account for $60 \%$ of the phase noise, and the switch transistors for the remaining $40 \%$. The distribution of the phase noise contributions can be regarded as typical for a well-designed differential VCO. Equally important is the value of the absolute contribution of the tank resistances to the phase noise; according to (9), the contribution of the single resistance should be $3.76 \cdot 10^{-16} \mathrm{~V}_{\mathrm{rms}}^{2} / \mathrm{Hz}$ at $3 \mathrm{MHz}$ frequency offset from the fundamental, which is only marginally lower than the simulated result. Further, the peak amplitude of the single-ended signal is $815 \mathrm{mV}$, very close to the value given by the expression $V_{\mathrm{p}}=(2 / \pi) R_{\mathrm{tank}}\left(2 I_{\text {bias }}\right)$, which assumes an ideal square waveform for the current into the tank. ${ }^{6}$ These results show that the simulated VCO is performing in a (quasi) optimal way, which is crucial, since we are next going to compare its phase-noise performance with those of the QVCOs previously mentioned. Finally, combining the data from noise and

\footnotetext{
${ }^{6}$ The factor $\left(2 I_{\text {bias }}\right)$ is due to the fact that we have defined $I_{\text {bias }}$ as the bias current for a single tank, and there are two tanks in the differential oscillator.
}

TABLE II

Simulated Noise POWER CONTRIBUtions (IN $V_{\mathrm{rms}}^{2} / H z$ ) AND PHASE NOISE AT $3 \mathrm{MHz}$ FREQUENCY OFFSET From THE 2-GHz FUNDAMENTAL FOR FOUR DIFFERENT $L C$-TANK QVCO DESIGNS $\left(Q_{\text {tank }}=10, I_{\mathrm{BIAS}}=4 \times 4 \mathrm{~mA}\right)$

\begin{tabular}{|c|c|c|}
\hline \multicolumn{3}{|c|}{ BS-QVCO } \\
\hline $\mathrm{R}_{\text {tank }}$ & $4 \times 9.10 \cdot 10^{-17}$ & $47.6 \%$ \\
\hline $\mathrm{M}_{s w}$ & $4 \times 6.81 \cdot 10^{-17}$ & $34.9 \%$ \\
\hline $\mathrm{M}_{c p l}$ & $4 \times 3.62 \cdot 10^{-17}$ & $18.5 \%$ \\
\hline Oscillation amplitude & \multicolumn{2}{|l|}{$800 \mathrm{mV}_{\mathrm{p}}$} \\
\hline Phase noise & \multicolumn{2}{|l|}{$-146.1 \mathrm{dBc} / \mathrm{Hz}$} \\
\hline \multicolumn{3}{|c|}{ TS-QVCO } \\
\hline $\mathrm{R}_{\text {tank }}$ & $4 \times 10.80 \cdot 10^{-17}$ & $59.8 \%$ \\
\hline $\mathrm{M}_{s w}$ & $4 \times 4.34 \cdot 10^{-17}$ & $24.1 \%$ \\
\hline $\mathrm{M}_{c p l}$ & $4 \times 2.90 \cdot 10^{-17}$ & $16.1 \%$ \\
\hline Oscillation amplitude & $790 \mathrm{mV}_{\mathrm{p}}$ & \\
\hline Phase noise & $-146.3 \mathrm{dBc} / \mathrm{H}$ & \\
\hline \multicolumn{3}{|c|}{ P-QVCO,$s_{c}=1 / 3$} \\
\hline $\mathrm{R}_{\text {tank }}$ & $4 \times 9.65 \cdot 10^{-17}$ & $43.3 \%$ \\
\hline $\mathrm{M}_{s w}$ & $4 \times 7.15 \cdot 10^{-17}$ & $32.0 \%$ \\
\hline $\mathrm{M}_{c p l}$ & $4 \times 5.53 \cdot 10^{-17}$ & $24.8 \%$ \\
\hline Oscillation amplitude & \multicolumn{2}{|l|}{$560 \mathrm{mV}_{\mathrm{p}}$} \\
\hline Phase noise & \multicolumn{2}{|l|}{$-142.4 \mathrm{dBc} / \mathrm{Hz}$} \\
\hline \multicolumn{3}{|c|}{$\mathrm{P}-\mathrm{QVCO}, s_{c}=1 / 2$} \\
\hline$\overline{\mathrm{R}_{\text {tank }}}$ & $4 \times 10.15 \cdot 10^{-17}$ & $40.5 \%$ \\
\hline $\mathrm{M}_{s w}$ & $4 \times 8.47 \cdot 10^{-17}$ & $33.7 \%$ \\
\hline $\mathrm{M}_{c p l}$ & $4 \times 6.47 \cdot 10^{-17}$ & $25.8 \%$ \\
\hline Oscillation amplitude & \multicolumn{2}{|l|}{$510 \mathrm{mV}_{\mathrm{p}}$} \\
\hline Phase noise & \multicolumn{2}{|l|}{$-141.1 \mathrm{dBc} / \mathrm{Hz}$} \\
\hline
\end{tabular}

oscillation amplitude, (8) gives a phase noise of $-143.8 \mathrm{dBc} / \mathrm{Hz}$ at $3 \mathrm{MHz}$ offset from the fundamental.

Turning now to the QVCOs, Table II summarizes the most important phase noise data. To repeat, these QVCOs were built around four $L C$ tanks identical to those used in the differential $\mathrm{VCO}$, while the bias current was doubled, so that the bias current per tank was the same in all situations. There is, however, one more degree of freedom in the design of a QVCO based on two differential VCOs: the strength of the coupling between the two differential VCOs [3]. While the phase error between the four phases can be reduced by increasing the coupling strength, an undesired result of this measure is that the phase noise increases as well. This trade off between phase noise and phase error is very strong in the P-QVCO [3], much less so in both the TSand BS-QVCO [2], [3]. Thus, in order to compare two different QVCO designs in a fair way, both phase error and phase noise must be taken into account. The coupling strength is quantified by the parameter $s_{c}$, defined as ${ }^{7}$

$$
s_{c}=\frac{W_{c p l}}{W_{s w}}
$$

where $W_{c p l}\left(W_{s w}\right)$ is the width of the coupling (switching) transistors, and the assumption has been made that all transistors have the same length. According to simulations (see also [2], [3]), the TS- and BS-QVCO optimized for minimum phase noise display the same phase error as the P-QVCO having a $s_{c}$

\footnotetext{
${ }^{7} s_{c}$ was called $\alpha$ in both [3] and [2]; in the present paper, the name has been changed in order to avoid confusion with the $\alpha$ appearing later in Appendix A.
} 
ranging from $1 / 2$ to $1 / 3$. The phase noise of the $\mathrm{P}-\mathrm{QVCO}$ was therefore simulated for these two values of $s_{c}$, while TS- and BS-QVCO were assigned a single $s_{c}$ value of five.

As clear from (10), if a QVCO performed exactly "equally well" as the differential VCO, we would expect a $3 \mathrm{~dB}$ lower phase noise compared to the case of the differential VCO. In fact, both BS- and TS-QVCO come very close to this limit, displaying a phase noise reduction of $2.3 \mathrm{~dB}$ and $2.5 \mathrm{~dB}$, respectively. It is worth noting that all QVCOs display a phase noise contribution from the single tank resistance very close to $9.4 \cdot 10^{-17} \mathrm{~V}_{\mathrm{rms}}^{2} / \mathrm{Hz}$, which is the (ideal) value yielded by (9), and that this value is very nearly $1 / 4$ of that for the differential oscillator, as expected. The performances of the P-QVCO, however, are sensibly poorer than the two S-QVCOs, displaying a phase noise between $3.7 \mathrm{~dB}$ and $5.2 \mathrm{~dB}$ higher. ${ }^{8}$ These results integrate those presented in [3], where it was shown that the TS-QVCO is much less sensitive to $1 / f$-noise upconversion into phase noise than the P-QVCO.

\section{A Systematic Source of Phase ERror}

As mentioned in [3], the implementation of the TS-QVCO there presented suffered from a sub-optimal layout, since the very long interconnections between the two center-tapped coils affected adversely the $Q$ of the tanks and the maximum achievable oscillation frequency. A new TS-QVCO design with optimized, very compact layout was therefore fabricated, and also here the phase error between the four phases was measured indirectly, as the image band rejection (IBR) in a single side-band (SSB) frequency up-converter, identical to the one used in [1]. To our surprise, the IBR data was this time much poorer, lying some 20-25 dB below those measured in [1].

Somewhat unexpectedly, this deteriorated IBR behavior could be traced back to the mutual inductance $M$ between the two center-tapped coils [16]. This is immediate to check by simulations, but can also be easily derived by using the linear model of the QVCO presented in [3], and reproduced in Fig. 2. Here $I_{Q B}$, which is in quadrature with $\mathrm{V}_{X}$, is generated by the large-signal coupling transconductance $\mathrm{G}_{M C}$, and $I_{I A}$, which is in phase with $V_{X}$, is generated by the large-signal switch transconductance $G_{M}$ (analogous relations apply to $I_{Q I}, I_{I B}$, and $\mathrm{V}_{Y}$ ). In the following, we will assume that the currents $I_{Q A}$ and $I_{Q B}$ are still in quadrature, despite the effect of the mutual inductance (this introduces only a negligible error on the final result). Straightforward circuit analysis [16] yields a departure from quadrature between $V_{X}$ and $V_{Y}$ by an angle $\delta$, given by

$$
\delta=2 \arctan \left(\frac{M}{L}\right)=2 \arctan (k)
$$

where $k$ is the coupling factor between the two coils. Thus, even if $k$ is as low as 0.01 , the phase error is already larger than $1^{\circ}$. Simulations on the TS-QVCO showed that the IBR was $35 \mathrm{~dB}$ at best for this value of $k$.

A brute-force solution to this problem is to diminish $k$ by increasing the distance between the two coils, accepting

\footnotetext{
${ }^{8}$ The performance deterioration for the P-QVCOs is mainly caused by the reduction of the effective $Q$ in the tanks [13], which results in smaller oscillation amplitudes (see Section II-A).
}

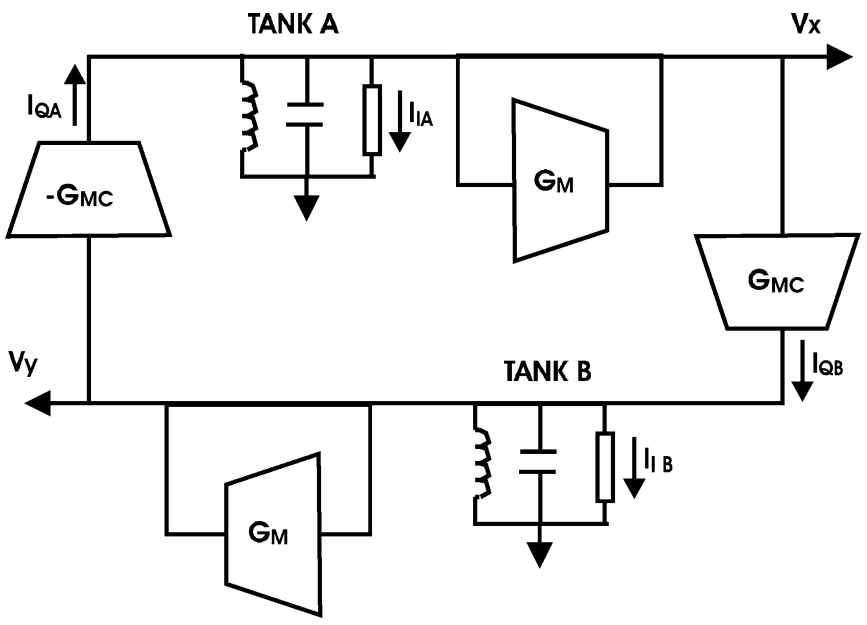

Fig. 2. Simplified linear model for a QVCO.

increased losses and increased parasitic capacitances. Basically, this would entail a retreat to the previous layout. A more appealing solution is to adopt a design having $k=0$ by construction, at least ideally. This can be achieved by replacing each center-tapped coil with two two-terminal coils, as shown in Fig. 3, where L1a and L1b belong to one differential VCO, $\mathrm{L} 2 \mathrm{a}$ and $\mathrm{L} 2 \mathrm{~b}$ to the other one. The direction of the current flowing into L1a and L1b, respectively, is such that the two magnetic fields generated have opposite directions (and equal magnitudes). Due to the symmetry of the layout, ideally the effects of the two fields on the inductors in the other oscillator (L2a and L2b) cancel out perfectly. The same is of course true of the effects of L2a and L2b on L1a and L1b. In practice, the mutual inductance between the tanks does not totally disappear, but is nevertheless strongly reduced. It can be noted that a mutual inductance also exists between coils belonging to the same VCO; however, it is easy to see that it has no impact on the IBR.

\section{Measurement Results}

\section{A. QVCOs With Two Center-Tapped Coils}

Several designs for both the TS- and BS-QVCO architectures have been fabricated in a standard $0.35 \mu \mathrm{m}$ CMOS process with three metal layers of thickness less than $1 \mu \mathrm{m}$ each. Extensive measurement results on a TS-QVCO have been reported in [3] and will not be repeated here.

A robust comparison between TS- and BS-QVCO has been obtained by building a BS-QVCO (Fig. 4) around the same $L C$ tanks as the TS-QVCO in [3], although such a layout is sub-optimal, as explained in Section IV. As a consequence, the estimated $Q$ at $2 \mathrm{GHz}$ is approximately six, while it was eight when the same tank was used in a (nonquadrature) differential VCO [17]. The center-tapped inductor had an estimated inductance value of approximately $2 \times 1.2 \mathrm{nH}$, and MOS devices working in accumulation/depletion were adopted as varactors. The whole tank capacitance was made of the parasitic capacitances of the transistors and of the metal routings. Table III shows dimensions and values for the various components in the BS-QVCO, which was optimized for phase-noise performances. 


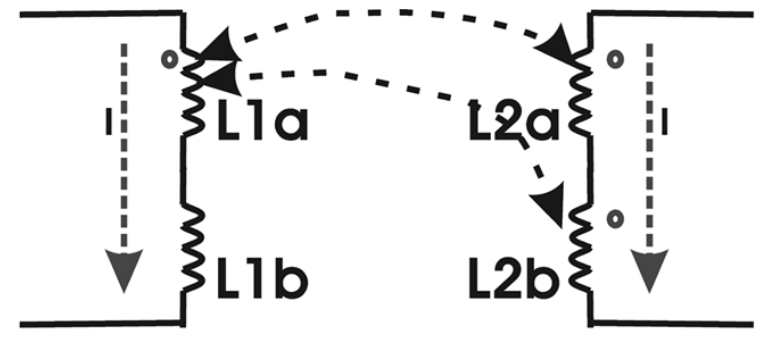

Lla

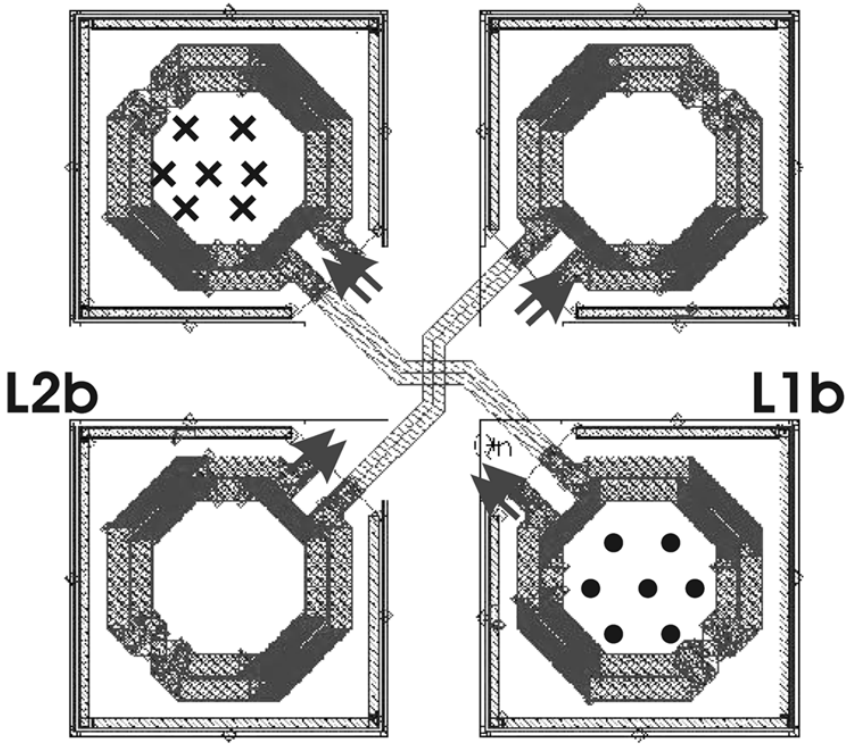

\section{$\longrightarrow$ Current in the inductor $X$ • Directions of the magnetic fields}

Fig. 3. Four-inductor design of the $L C$ tanks. The sum of the magnetic fields generated by L1a and L1b is ideally zero at each of the two inductors L2a and L2b (and vice versa).

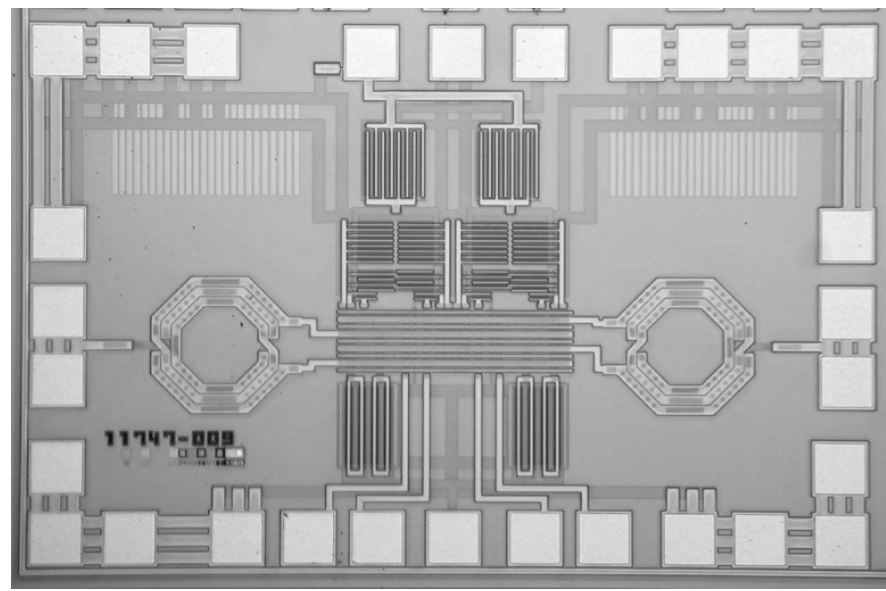

Fig. 4. Die photograph of the BS-QVCO $(1.4 \mathrm{~mm} \times 0.9 \mathrm{~mm})$.

All measurements on the BS-QVCO have been performed with a power supply as low as $1.3 \mathrm{~V}$, for a current consumption of $16 \mathrm{~mA}$. The QVCO could be tuned from $1.91 \mathrm{GHz}$ to $2.27 \mathrm{GHz}$, for a tuning range of $17 \%$. The phase noise at $3 \mathrm{MHz}$ offset frequency from the carrier was $-140 \mathrm{dBc} / \mathrm{Hz}$ or lower across the tuning range (Fig. 5). Fig. 6 shows the phase-noise
TABLE III

DIMENSIONS AND VALUES FOR THE BS-QVCO

\begin{tabular}{lr}
\hline \hline & Transistors \\
$\mathrm{M}_{c p l}$ & $400 \mu \mathrm{m} \times 0.35 \mu \mathrm{m}$ \\
$\mathrm{M}_{s w}$ & $800 \mu \mathrm{m} \times 0.35 \mu \mathrm{m}$ \\
$\mathrm{M}_{\text {varactor }}$ & $1200 \mu \mathrm{m} \times 0.35 \mu \mathrm{m}$ \\
$\mathrm{M}_{\text {src }}$ & $2000 \mu \mathrm{m} \times 1.0 \mu \mathrm{m}$ \\
\hline \multicolumn{2}{c}{ Reactors } \\
$\mathrm{L}_{\text {tank }}$ & $\approx 2.4 \mathrm{nH}$ \\
$\mathrm{Q}$ of the LC-tank $\approx 6$ at $2.0 \mathrm{GHz}$ \\
\hline \hline
\end{tabular}

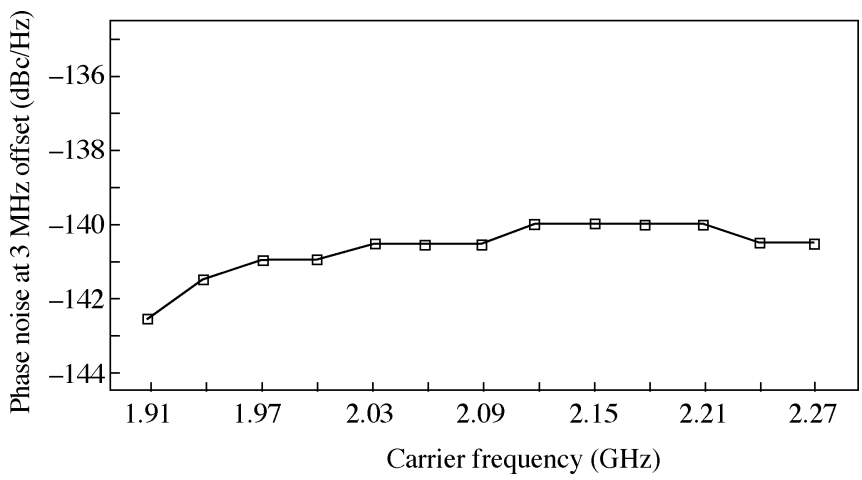

Fig. 5. Measured phase noise for the $\mathrm{BS}-\mathrm{QVCO}$ at $3 \mathrm{MHz}$ offset frequency.

plot for the highest oscillation frequency. According to (11), and using the data in Fig. 5, the minimum phase-noise FoM across the tuning range is $184 \mathrm{~dB}$, which is no less than $6 \mathrm{~dB}$ higher than the minimum FoM displayed by the TS-QVCO in [1] (approximately $2 \mathrm{~dB}$ are accounted for by the lower power supply, and one more $\mathrm{dB}$ is due to the fact that the tuning range for the TS-QVCO was shifted some $200 \mathrm{MHz}$ down in frequency, which resulted in a slightly lower $L C$-tank $Q$ at the lowest oscillation frequencies). This comparison shows that BS-QVCO is capable, at least in these experiments, to behave closer to the ideal case than the TS-QVCO. In fact, substituting the relevant data in (6), and assuming that the tank resistances are responsible for approximately $50 \%$ of the phase noise generation (see Table II), the calculated phase noise is at most only $1 \mathrm{~dB}$ lower than measured (the main uncertainty in this comparison being the exact value of the tank-Q).

Another interesting comparison is that between the phasenoise FoM for the BS-QVCO and that for the differential VCO presented in [17], which covered approximately the same frequency range, and whose $L C$ tank (based on exactly the same center-tapped inductor) had a $Q$ of eight at $2 \mathrm{GHz}$. This VCO has a minimum FoM of $183 \mathrm{~dB}$, which is $1 \mathrm{~dB}$ lower than the minimum FoM for the BS-QVCO. This is even more remarkable considering that the VCO in [17] made use of two noise reduction techniques, the on-chip noise filter [18] and the off-chip inductive degeneration of the tail transistor [17], which greatly enhanced its FoM. For the BS-QVCO it has been checked that the noise filter (implemented in a second, otherwise identical QVCO design) does not lead to an increase of the minimum FoM, while inductive degeneration increases it by $1 \mathrm{~dB}$ at most, too modest an improvement to grant the use of an external component. 


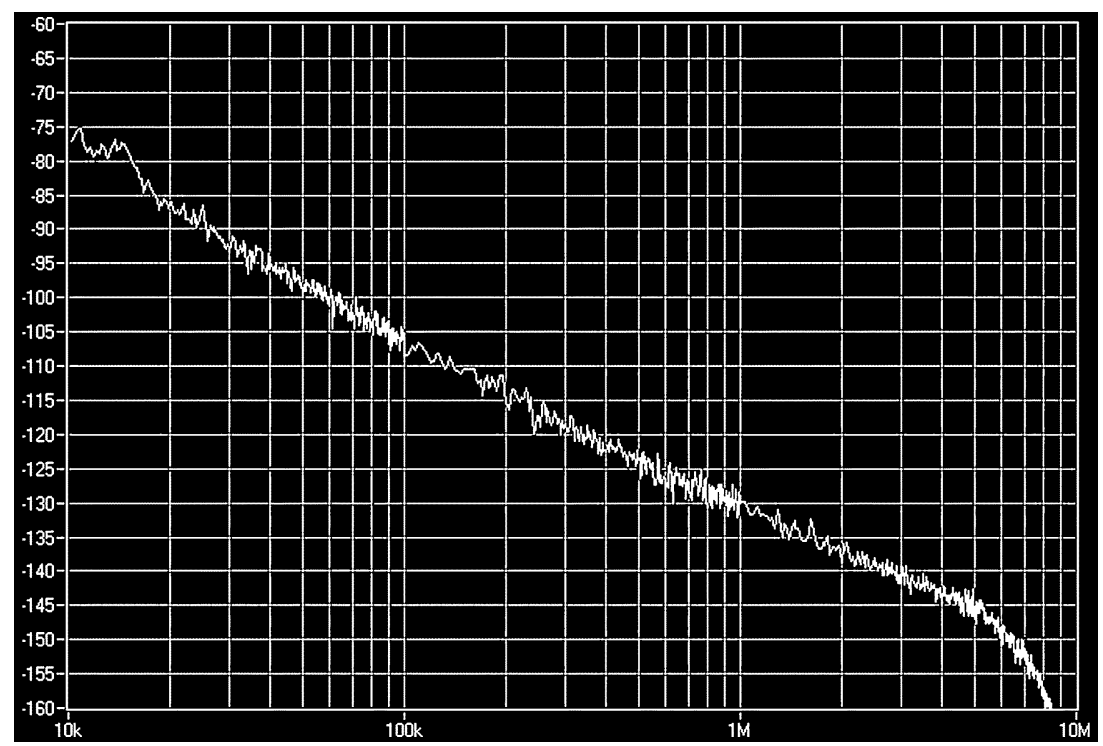

Fig. 6. Measured phase noise for the BS-QVCO at $2.27 \mathrm{GHz}$ oscillation frequency.

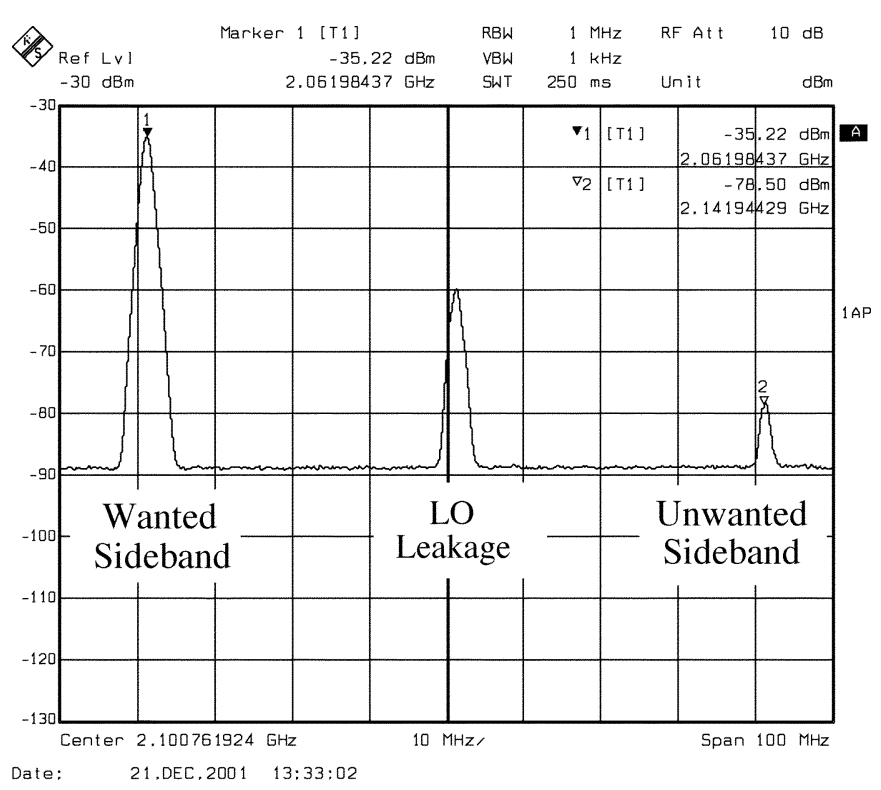

Fig. 7. Upconverted baseband signals and LO leakage for the BS-QVCO at 2.1-GHz carrier frequency $(\mathrm{IBR}=43 \mathrm{~dB}$, minimum measured value across the tuning range).

As mentioned in Section IV, the phase error is measured as the IBR in an SSB up-converter, identical to the one used in [1]. Thus, the IBR is comprehensive not only of the mismatches in the QVCO, but also of those in the SSB-mixers and in the 4-stage $R C$ polyphase filter used to generate the quadrature baseband signals. In all five measured samples the IBR is $50 \mathrm{~dB}$ or higher at the lower oscillation frequencies, and decreases rather sensibly with increasing oscillation frequencies, probably indicating that varactor mismatches are the dominant cause for the phase error. Fig. 7 shows the minimum IBR (43 dB) measured for these samples. Assuming that the IBR is entirely caused by a deviation from quadrature of otherwise ideal sinusoidal outputs, simulations for the upconverter indicate that an IBR of $43 \mathrm{~dB}$ is equivalent to a phase error of approximately $0.6^{\circ}$ between the $I$ and $Q$ phases. As expected

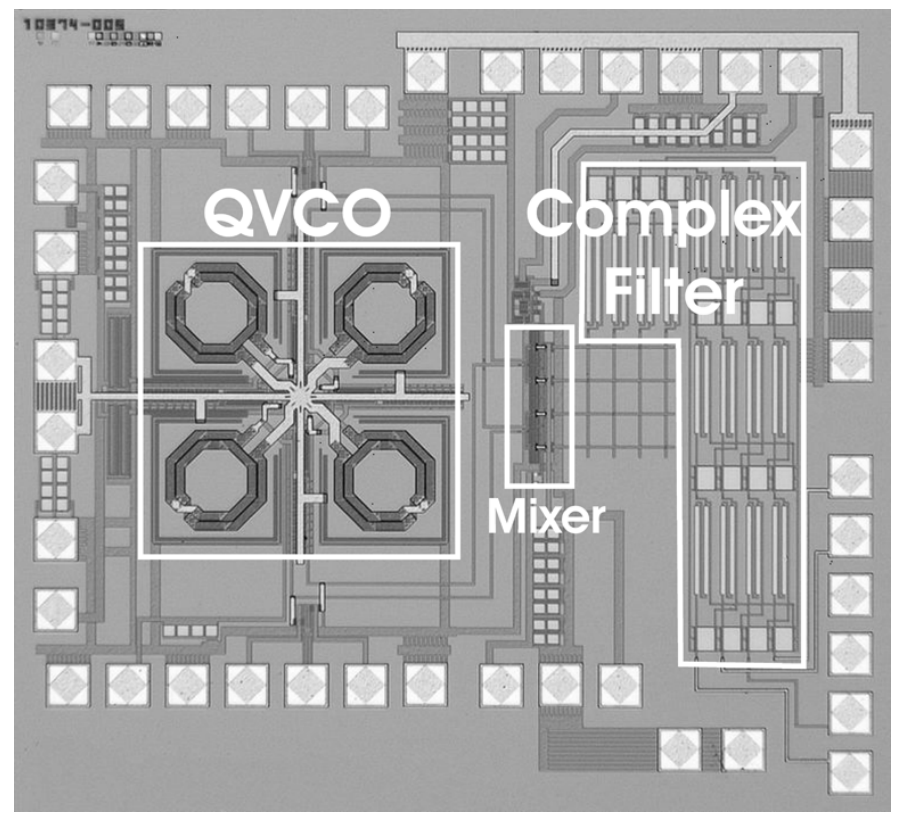

Fig. 8. Chip photograph of the new TS-QVCO, together with the SSB upconversion circuits $(1.5 \mathrm{~mm} \times 1.8 \mathrm{~mm})$.

from IBR simulations [2], this phase error is larger than the $0.25^{\circ}$ measured for the TS-QVCO.

\section{B. QVCOs With Four Coils}

As discussed in Section IV, both circuit analysis and simulations indicate that a parasitic mutual inductance between the tanks of the coupled VCOs is a major cause of phase error in a QVCO. In order to measure directly the impact of the mutual inductance on the phase error, two new four-coil TS-QVCOs were designed, identical in all respects, except that in one of them (referred to as TS-swap) the directions of two magnetic fields were reversed, so that the magnetic coupling between the two differential VCOs was doubled instead of nulled. Fig. 8 shows the die photo of the new TS-QVCO, whose layout is now highly 


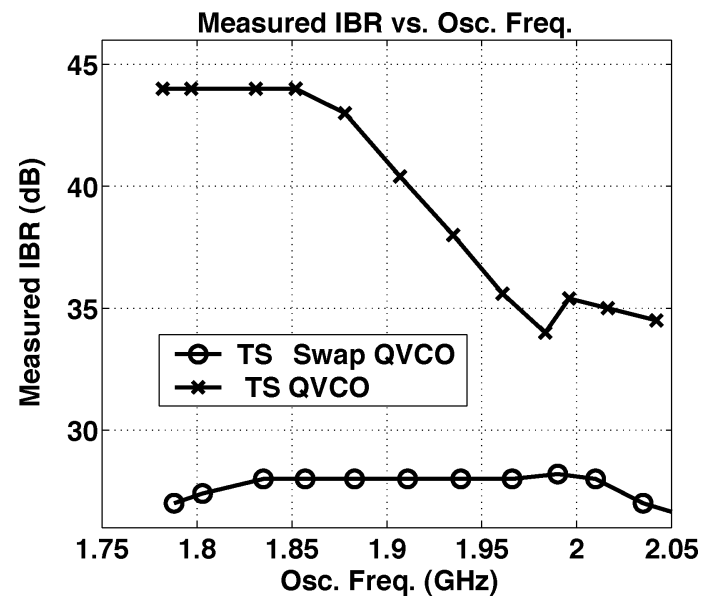

Fig. 9. Measured IBR for the TS- and TS-swap QVCOs across the tuning range.

quad-symmetrical. ${ }^{9}$ The four inductors are placed as close as possible to each other, in order to minimize the loss caused by the connections. Four metal layers of standard thickness were now available in the same basic CMOS process previously used, and the $1.1 \mathrm{nH}$ inductors made use of the top three layers. The inductor Q, simulated following the theory in [20], was nine at $2 \mathrm{GHz}$.

Fig. 9 shows the measured IBRs for both a TS- and a TS-swap QVCO, where the data is representative for all measured specimens. At low oscillation frequencies, the TS-QVCO presents an IBR as much as $17 \mathrm{~dB}$ higher than that for the TS-swap QVCO, which proves that the mutual inductance effect really is the dominating cause of phase error in the TS-swap design. The IBR drop at higher oscillation frequencies for the TS-QVCO is again very likely due to the accumulation-mode varactors, which obviously match poorly when working in the depletion region; this should be easily remedied by adopting a mixedmode tuning approach, which allows for smaller continuoustuning devices. The equivalent phase error is at most approximately $1^{\circ}$ across the tuning range. We can add that the best IBR for this TS-QVCO is some $8 \mathrm{~dB}$ lower than the IBR for the TS-QVCO in [1], which probably indicates that the phase error in the new TS-QVCO is actually still dominated by the residual mutual inductive coupling between the two inductor pairs.

Finally, the phase noise for the TS-QVCO was $-140 \mathrm{dBc} / \mathrm{Hz}$ at $3 \mathrm{MHz}$ frequency offset, for a current consumption of $16 \mathrm{~mA}$ from a $2 \mathrm{~V}$ power supply, resulting in a phase-noise FoM of 182 $\mathrm{dBc} / \mathrm{Hz}$. This is as much as $4 \mathrm{~dB}$ higher than the FoM of the TS-QVCO in [1], although the comparison is slightly biased by the fact that a fourth metal layer was available in the second experiment.

\section{CONCLUSION}

This paper has presented an analytical study of the phase noise in multiphase $L C$ tank oscillators, deriving explicit phase-noise formulas for the noise contributions of the tank losses. As a result, the comparison between different VCO im-

\footnotetext{
${ }^{9}$ A quad-symmetric layout has already been proposed in [19], where the effect of the parasitic magnetic coupling was not treated. The symmetry of the layout must be such that this effect is suppressed, as explained above.
}

plementations, and even between VCOs with different numbers of phases, can be made with little additional effort.

The analysis has made use of Hajimiri's phase-noise theory in its closed-form formulation, and a proof of the correctness of the approach was also supplied.

The newly derived results, together with spectreRF simulations, were used to assess the performances of two quadrature VCOs, referred to as TS-QVCO and BS-QVCO, which were found to possess excellent capabilities. A $0.35 \mu \mathrm{m}$ CMOS prototype for a 2-GHz BS-QVCO showed a phase error of $0.6^{\circ}$ and a phase noise of $-140 \mathrm{dBc} / \mathrm{Hz}$ or less at $3 \mathrm{MHz}$ offset frequency from the carrier, for a current consumption of $16 \mathrm{~mA}$ from a 1.3 $\mathrm{V}$ power supply, resulting in a phase-noise FoM of $184 \mathrm{dBc} / \mathrm{Hz}$. This is close to the maximum FoM that can be expected in the technology used.

Phase-error measurements on later QVCO designs suggested that the parasitic mutual inductive coupling between the two center-tapped coils in the QVCOs could play a dominant role in the generation of the phase error. This hypothesis was confirmed by data taken on two more TS-QVCO prototypes, where a technique for greatly reducing this cause of phase error was also adopted with success. As a results, more compact layouts are feasible, resulting in higher quality factors for the $L C$ tanks, and higher oscillation frequencies.

\section{APPENDIX A}

In the following, we will show that in a multiphase $L C$-tank oscillator the phase noise generated by the noise current source in each tank is very well approximated by (6), when $\Gamma$ is given by (4). To do so, we follow the approach by Kärtner [5], who studied the deviations from the stable limit cycle in the oscillator caused by stochastic noise sources. The reader is of course referred to [5] for the complete theory; in the following (14)-(22), taken directly from [5], the original notation is conserved.

The oscillator is a dynamic system described by the system of nonlineal differential equations

$$
\dot{\vec{x}}=\vec{F}(\vec{x}, t, \vec{\xi})
$$

where $\vec{x}$ is the vector of state variable (i.e., voltages across capacitors and currents through inductors), and $\vec{\xi}$ is the vector of the noise sources.

If the noise sources are white, we can expand (14) linearly in $\vec{\xi}$ as

$$
\dot{\vec{x}}=\vec{F}(\vec{x}, \overrightarrow{0})+\mathrm{G}(\vec{x}) \vec{\xi}
$$

where

$$
G_{i j}(\vec{x})=\left.\frac{\partial F_{i}(\vec{x}, \vec{\xi})}{\partial \xi_{j}}\right|_{\vec{\xi}=\overrightarrow{0}}
$$

This approximation of (14) is justified by the smallness of the noise sources. We now assume that the noise sources cause $\vec{x}$ to deviate from the stable limit cycle $\vec{x}^{0}$, and we can write

$$
\vec{x}(t)=\vec{x}^{0}(t+\theta(t))+\Delta \vec{x}_{\perp}(t+\theta(t))
$$

where $\theta$ is the excess phase and $\Delta \vec{x}_{\perp}$ is the deviation perpendicular to the limit cycle. 
Defining $y=t+\theta(t)$, it can be shown that

$$
\dot{\vec{\theta}}(t)=\alpha(y) \vec{\xi}(t(y))+\beta(y) \Delta \vec{x}_{\perp}(y)
$$

where

$$
\begin{aligned}
& \alpha(y)=\frac{\vec{n}^{T}(y) G(y)}{\left|\vec{x}^{0 \prime}(y)\right|}, \\
& \beta(y)=\frac{\vec{n}^{T}(y) D F\left(\vec{x}^{0}(y)\right)+\vec{n}^{T \prime}(y)}{\left|\vec{x}^{0 \prime}(y)\right|},
\end{aligned}
$$

with

$$
\begin{aligned}
\vec{n}(t) & =\frac{\dot{\vec{x}}^{0}(t)}{\left|\dot{\vec{x}}^{0}(t)\right|}, \\
D F\left(\vec{x}^{0}(y)\right)_{i j} & =\left.\frac{\partial F_{i}(\vec{x})}{\partial x_{j}}\right|_{\vec{x}=\vec{x}^{0}(y)}
\end{aligned}
$$

The prime symbol at the exponent denotes derivation with respect to the argument. The term in $\alpha$ in (18) yields ultimately the excess phase generated by the noise sources acting in the direction parallel to the limit cycle, which is the same as the excess phase given by (1). The term in $\beta$, on the other hand, gives the excess phase generated by noise sources orthogonal to the limit cycle, of which (1) takes no account. While the sum of the two contributions from the $\alpha$ and $\beta$ terms is constant (the concept of excess phase would not have any physical meaning otherwise), the individual contributions are not constant in the general case, but are dependent on the state variable used. The problem can also be reformulated in such a way that the excess phase is independent of the "orthogonal" noise sources [6].

We will show next that the state variables in an $L C$-tank oscillator can be chosen in such a way that $\beta$ almost vanishes, so that the only significant causes of excess phase are the noise sources acting in a direction parallel to the limit cycle, and therefore (1) is applicable.

\section{A. Calculations on a Simple LC-Tank Oscillator Model}

We will treat here the case of a single $L C$-tank oscillator for simplicity, but it will be clear that the approach is readily extended to the general case. With reference to Fig. 10, and choosing as state variables the voltage across the capacitor and the current flowing into the inductor, we obtain:

$$
\begin{aligned}
\dot{v}_{C} & =\frac{1}{C}\left[-i_{L}-\frac{1}{R} v_{C}+i\left(v_{C}\right)\right], \\
\dot{i}_{L} & =\frac{1}{L} v_{C} .
\end{aligned}
$$

We now normalize (24) by multiplication with the impedance $\sqrt{L / C}$, obtaining the equation

$$
\dot{v}_{L} \equiv \sqrt{\frac{L}{C}} \dot{i}_{L}=\frac{1}{\sqrt{L C}} v_{C}=\omega_{0} v_{C}
$$

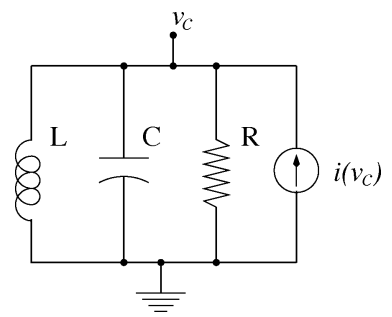

Fig. 10. Simple model of single-ended $L C$-tank oscillator.

so that the new state equation system becomes

$$
\begin{aligned}
\dot{v}_{C} & =-\omega_{0} v_{L}+\frac{1}{C}\left[-\frac{1}{R} v_{C}+i\left(v_{C}\right)\right], \\
\dot{v}_{L} & =\omega_{0} v_{C} .
\end{aligned}
$$

We proceed by noting that if the $Q$ of the tank is even moderately high, the oscillation at the tank output is, with very good approximation, a sinusoid with angular frequency $\omega_{0}$; therefore, with arbitrary phase for $v_{C}(t)$, we can write

$$
v_{C}(t)=A \cos \left(\omega_{0} t\right)
$$

where $A$ is the amplitude of the oscillation. The expression for $v_{L}(t)$ follows immediately from (27) and (28):

$$
v_{L}(t)=A \sin \left(\omega_{0} t\right) .
$$

The crucial result yielded by normalization (25) is that $v_{L}(t)$ has the same amplitude as $v_{C}(t)$.

Identifying now $x_{1}(t)$ with $v_{C}(t)$ and $x_{2}(t)$ with $v_{L}(t)$, we obtain from (21)

$$
\vec{n}^{T}(t)=\left[-\sin \left(\omega_{0} t\right), \cos \left(\omega_{0} t\right)\right]
$$

and from (22)

$$
D F=\left(\begin{array}{cc}
-\frac{1}{R C}+\frac{1}{C} \frac{\partial i\left(v_{C}\right)}{\partial v_{C}} & -\omega_{0} \\
\omega_{0} & 0
\end{array}\right)
$$

We are now ready to calculate the two components of the vector appearing at the numerator of $\beta(y)$ :

$$
\begin{aligned}
& \left(\vec{n}^{T}(y) D F\left(\vec{x}^{0}(y)\right)+\vec{n}^{T \prime}(y)\right)_{1} \\
& =-\left[-\frac{1}{R C}+\frac{1}{C} \frac{\partial i\left(v_{C}\right)}{\partial v_{C}}\right] \sin \left(\omega_{0} y\right) \\
& \quad+\omega_{0} \cos \left(\omega_{0} y\right)-\omega_{0} \cos \left(\omega_{0} y\right) \\
& =\left[\frac{1}{R C}-\frac{1}{C} \frac{\partial i\left(v_{C}\right)}{\partial v_{C}}\right] \sin \left(\omega_{0} y\right) \\
& \left(\vec{n}^{T}(y) D F\left(\vec{x}^{0}(y)\right)+\vec{n}^{T \prime}(y)\right)_{2} \\
& \quad=\omega_{0} \sin \left(\omega_{0} y\right)-\omega_{0} \sin \left(\omega_{0} y\right)=0
\end{aligned}
$$

Thus, we see from the above equations that the large terms in $\omega_{0}$ cancel out, and it is elementary although tedious to check that this happens only if the normalization (25) is used. In general, (32) is not identically zero, since the two terms $1 / R$ and $\partial i\left(v_{C}\right) / \partial v_{C}$ do not cancel each other, not even averaged across an oscillation period; in fact, the term $-\partial i\left(v_{C}\right) / \partial v_{C}$ cannot 

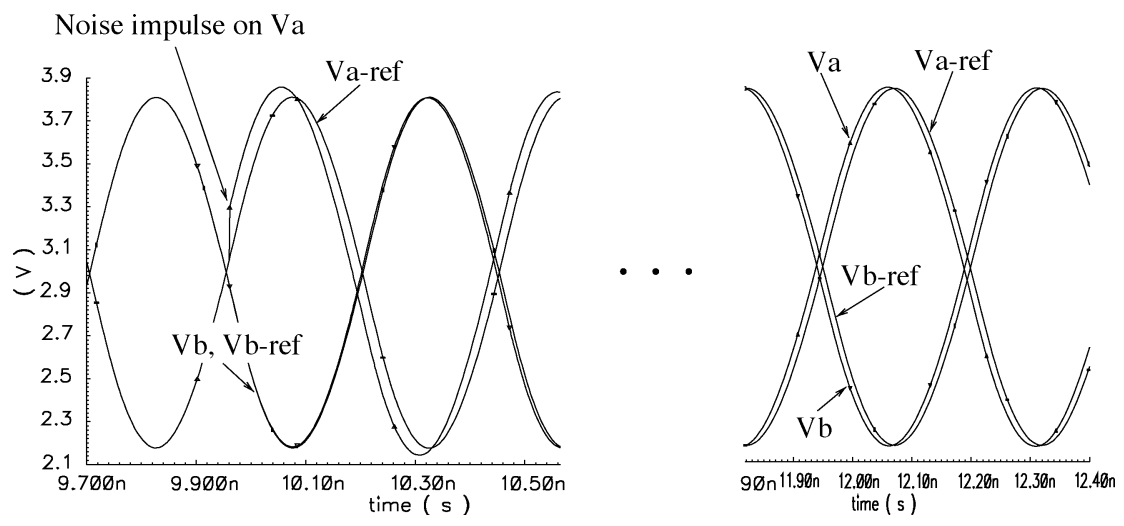

Fig. 11. Changes on the relative phases of the two steady-state phase-opposed waveforms $\mathrm{V}_{a}$ and $\mathrm{V}_{b}$, in presence of a single-ended noise impulse on $\mathrm{V}_{a} . \mathrm{V}_{a-r e f}$ and $\mathrm{V}_{b-r e f}$ are the waveforms when the noise impulse is removed.

be just a constant negative conductance, since it must include the nonlinear drop in conductance for large values of $v_{C}(t)$, essential for limiting the oscillation amplitude in any real-life oscillator. At any rate, spectreRF simulations show that the remaining excess phase contribution from $\beta$ in (18) is negligible, compared to the term in $\alpha$, already for a moderate $L C$-tank $\mathrm{Q}$, a fact that renders the phase noise caused by the tank resistance remarkably independent of the nature of the loss compensation in the oscillator. At the same time, it is immediate to show that the very normalization (25) results in (4) (with $n=1$ for the example treated here), which is the sought result.

Lest it might be thought that the above proof is superfluous, it can be easily checked that (1) yields wrong results in general, when applied to the circuit in Fig. 10 with the tank inductance replaced by a gyrator-capacitor circuit, i.e., when

$$
L=\frac{C_{L}}{g_{m 1} g_{m 2}}
$$

where the particular values of capacitance $C_{L}$, and of gyrator transconductances $g_{m 1}$ and $g_{m 2}$, are arbitrary, as long as the value of $L$ is kept constant. The voltage $v_{C_{L}}$ across $C_{L}$ is now the second state variable of the circuit, and it is easy to check that $v_{C_{L}}$ has a peak value dependent on the ratio of $g_{m 1}$ to $g_{m 2}$. Therefore, $\Delta \phi$ in (1) comes to depend on this ratio, which is wrong, since the way $L$ is implemented cannot affect the phase noise generated by $R$ (the skeptical reader can easily check this through numerical simulations).

\section{APPENDIX B}

The purpose of this appendix is to give an intuitive proof of the fact that the phase noise of an oscillator is independent of the node, or combination of nodes, where it is measured [12].

Once again, we make use of the ISF theory, where the phase noise is modeled as an excess phase, introduced in the oscillation by a noise source. As long as this is true, it is immediate to realize that the phase noise is independent of the circuital node where the phase noise is measured, since at steady state all voltages and currents in an oscillator are linked by well-defined and constant phase relations. As an example, we can see that the two signal $V_{a}$ and $V_{b}$ in Fig. 11 recover the steady-state phase-opposition relation a short time after the appearance of the noise impulse on signal $V_{a}$. This means that the noise impulse has caused the same excess phase in both signals, which in turn implies that they display the same phase noise. It should be noted that this is true even if the two signals have different amplitudes, since the excess phase is the same for both, independently of the respective amplitudes (if this result sounds incorrect, it may be considered that the carrier-to-sideband ratio in a frequency-modulated sinusoid is only dependent on the modulation index, and independent of the amplitude of the sinusoid). However, it should not be concluded from this that all nodes are affecting the phase noise performance in the same way, since the generation of phase noise may be dominated by only one or a few of them; what is true is that the generated phase noise affects all nodes in the same way.

Another way of describing the above result is the following: the excess phase detected at one node is perfectly correlated to the excess phase detected at any other node. Thus, whether the phase noise is measured single-ended, or differentially, or even "quad-differentially," both signal power and noise power are increased by the same factor, leaving the phase noise unaltered.

\section{ACKNOWLEDGMENT}

P. Andreani would like to thank his friend M. Hvala for inspiring conversations.

\section{REFERENCES}

[1] P. Andreani, "A low-phase-noise, low-phase-error $1.8 \mathrm{GHz}$ quadrature CMOS VCO," in IEEE ISSCC Dig. Tech. Papers, Feb. 2002, pp. 290-291.

[2] — " "A $2 \mathrm{GHz}, 17 \%$ tuning range quadrature CMOS VCO with high figure-of-merit and 0.6 $6^{\circ}$ phase error," in Proc. ESSCIRC, Sept. 2002.

[3] P. Andreani, A. Bonfanti, L. Romanò, and C Samori, "Analysis and design of a $1.8 \mathrm{GHz}$ CMOS LC quadrature VCO," IEEE J. Solid-State Circuits, vol. 37, pp. 1737-1747, Dec. 2002.

[4] A. Hajimiri and T. H. Lee, "A general theory of phase noise in electrical oscillators," IEEE J. Solid-State Circuits, vol. 33, pp. 179-194, Feb. 1998

[5] F. X. Kärtner, "Determination of the correlation spectrum of oscillators with low phase noise," IEEE Trans. Microwave Theory Tech., vol. 37, pp. 90-101, Jan. 1989.

[6] _ "Analysis of white anf $f^{-\alpha}$ noise in oscillators," Int. J. Circuit Theory Applicat., vol. 18, pp. 485-519, 1990.

[7] A. Demir, A. Mehrotra, and J. Roychowdhury, "Phase noise in oscillators: A unifying theory and numerical methods for characterization," IEEE Trans. Circuits Syst. I, vol. 47, pp. 655-674, May 2000. 
[8] G. Coram, "A simple 2-D oscillator to determine the correct decomposition of perturbations into amplitude and phase noise," IEEE Trans. Circuits Syst. I, vol. 48, pp. 896-898, July 2001.

[9] Affirma RF Simulator User Guide, Cadence Product Documentation, version 4.4.6, Apr. 2001

[10] A. Hajimiri, S. Limotyrakis, and T. H. Lee, "Jitter and phase noise in ring oscillators," IEEE J. Solid-State Circuits, vol. 34, pp. 790-804, June 1999.

[11] A. Hajimiri and T. H. Lee, "Corrections to "A general theory of phase noise in electrical oscillators"," IEEE J. Solid-State Circuits, vol. 33, p. 928, June 1998.

[12] A. Demir and A. Sangiovanni-Vincentelli, Analysis and Simulation of Noise in Nonlinear Electronic Circuits and Systems. Boston, MA: Kluwer, 2000, ch. 6.

[13] J. van der Tang, P. van de Ven, D. Kasperkovitz, and A. van Roermund, "Analysis and design of an optimally coupled 5-GHz quadrature LC oscillator," IEEE J. Solid-State Circuits, vol. 37, pp. 657-661, May 2002.

[14] A. Rofougaran, J. Rael, M. Rofougaran, and A. Abidi, "A $900 \mathrm{MHz}$ CMOS LC-oscillator with quadrature outputs," in IEEE ISSCC Dig. Tech. Papers, Feb. 1996, pp. 392-393.

[15] F. Behbahani et al., "A fully integrated low-IF CMOS GPS radio with on-chip analog image rejection," IEEE J. Solid-State Circuits, vol. 37, pp. 1721-1727, Dec. 2002.

[16] X. Wang and P. Andreani, "Impact of mutual inductance and parasitic capacitance on the phase-error performance of CMOS quadrature VCOs," in Proc. ISCAS, vol. 1, May 2003, pp. 661-664.

[17] P. Andreani and H. Sjöland, "Tail current noise suppression in RF CMOS VCOs," IEEE J. Solid-State Circuits, vol. 37, pp. 342-348, Mar. 2002.

[18] E. Hegazi, H. Sjöland, and A. Abidi, "A filtering technique to lower LC oscillator phase noise," IEEE J. Solid-State Circuits, vol. 36, pp. 1921-1930, Dec. 2001.

[19] P. Vancorenland and M. Steyaert, "A $1.57 \mathrm{GHz}$ fully integrated very low phase noise quadrature VCO,” IEEE J. Solid-State Circuits, vol. 37, pp. 653-656, May 2002.

[20] Y. Cao et al., "Frequency-independent equivalent-circuit model for on-chip spiral inductors," IEEE J. Solid-State Circuits, vol. 38, pp. 419-426, Mar. 2003.

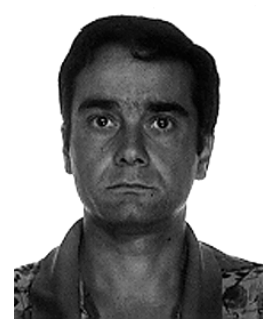

Pietro Andreani (S'98-A'99-M'01) received the M.S.E.E. degree from the University of Pisa, Italy, in 1988.

He joined the Department of Applied Electronics, Lund University, Sweden, in 1990, where he contributed to the development of software tools for digital ASIC design. After working at the Department of Applied Electronics, University of Pisa, as a CMOS IC Designer during 1994, he rejoined the Department of Applied Electronics, Lund University, as an Associate Professor, where he was responsible for the analog IC course package between 1995 and 2001, and where he received the Ph.D. degree in 1999. He is currently a Professor at the Center for Physical Electronics, Ørsted DTU, Technical University of Denmark, Kgs. Lyngby, Denmark, with analog/RF CMOS IC design as his main research field.

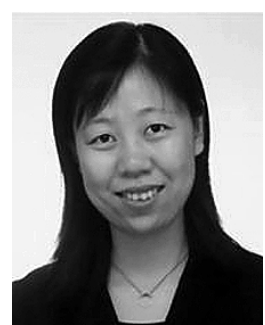

Xiaoyan Wang was born in China in 1976. She received the Bachelor degree from Nanjing University, China, in 1997, and the Master degree from the $\mathrm{Na}-$ tional University of Singapore in 2001.

In 2002, she joined the Center for Physical Electronics, Ørsted DTU, Technical University of Denmark, where she is currently a Ph.D. candidate working on integrated RF VCOs. 